\title{
Influence of soybean phytoestrogens exposure on the Mouse Stem cells differentiation
}

\author{
El-Mahdy, T.O.M. ${ }^{1}$; El-Nahla, S.M.M. ${ }^{1}$; Takahashi, S. ${ }^{2,3,4}$; Basha, W.A. ${ }^{1,2,3}{ }^{*}$ \\ ${ }^{1}$ Department of Anatomy and Embryology, Faculty of Veterinary Medicine, Suez Canal \\ University, Ismailia, Egypt. \\ ${ }^{2}$ Laboratory Animal Resource Center, University of Tsukuba, Tsukuba, Ibaraki, Japan. \\ ${ }^{3}$ Department of Anatomy and Embryology, Graduate School of Comprehensive Human \\ Sciences, University of Tsukuba, Tsukuba, Ibaraki, Japan. \\ ${ }^{4}$ International Institute for Integrative Sleep Medicine (WPI-IIIS), University of Tsukuba, \\ Tsukuba, Ibaraki, Japan.
}

Received September, accepted September 2018

\section{Abstract}

The toxic effects and mechanism of soy isoflavones such as genistein and diadzein on the early embryonic development and differentiation are still unknown. We aimed to elucidate the developmental and differentiation alterations occurred after either single exposure or co-exposure of soy isoflavones (Genistein and Diadzein) on the mEScs in order to understand the effects and possible pathways of phytoestrogen treatments on the early embryonic development. In the pre-sent study, mouse embryonic stem cells (mEBs) differentiate when exposed to different concentrations of genistein and/or diadzein for 5 days and their proliferation, apoptosis and differentiation capacities were evaluated using RT-qPCR analysis. Our results confirmed that soy phytoestrogens had differential effects on regulation of proliferation and apoptosis and the differentiation ability of the mouse stem cells at different levels varies from increase in the differentiation markers of the three germ layers or only ectoderm markers together with up regulation of Oct4 and Rex 1 transcription factors and in some cases with down regulation of Nanog in comparison with the control condition and these variations depend on the concentration and whether single or co-treatment of these soy phytoestrogens were used. All of these effects appeared to be related to down regulation effect of Esrrb. Our entire result concluded that soy isoflavones might disturb differentiation of mEBs and these phytochemicals may function via the estrogen related receptor $\beta$ (ESRR $\beta$ )-mediated pathway. 
Keywords: Phytoestrogens, Differentiation, Embryonic Stem cell, ESRRß.

\section{Introduction}

Chemicals in our foods cause severe problems throughout pregnancy especially in the early critical developmental phase while the main body systems of the fetus are developing (Barouki et al., 2012). About $10 \%$ of the birth defects are due to the exposure of mothers to the teratogenic agents during pregnancy. So, it becomes essential to evaluate the toxicological safety of any phytochemicals consumed by pregnant women and it is important to focus carefully on the deleterious molecular changes induced by these agents (Vandenberg et al., 2002). Development of rat embryo is affected by teratogens mostly during the process of organogenesis, which is recognized as the time period from the occurrence of the neural plaque to the closure of the plate. This period starts on the 6th day and going on for 10 days (Barouki et al., 2012).

Recently, Embryonic Stem Cells (EScs) are considered a useful model to investigate the processes of embryogenesis and differentiation as this process is similar to that occurred within the intact blastocyst. Some protocols are established for differentiation of mouse embryonic stem cells (mES) depending on the formation of embryoid bodies (EBs) to initiate formation of the three germ lineages: endoderm, ectoderm and mesoderm. During EB formation in vitro, only a small fraction of differentiated definitive endodermal cells arise while a predominance of ectodermal and mesodermal cells is generated (Martin, 1981). These procedures based on the ability of Mouse ES cells to remain undifferentiated when cultured in media containing serum and leukemia inhibitory factor (LIF), a cytokine while, induction of the Es cell differentiation into an embryoid body, a three-dimensional mass of cells of the various lineages resembling an early embryo, was reported to occurred in case of culture of Es cells without LIF (Zandstra et al., 2000). Proceeding from this point, novel test systems based on embryonic stem cell (ESC) tests are widely used in various areas of in vitro toxicology and biomedicine, and considered as future alternatives to animal testing together with using different assessment methods such as measuring the expression of marker genes that expressed during the differentiation of the ES cell into an embryoid body, where it helps evaluating the effects occurred to the embryonic stem cells and the developing embryoid bodies which in turn improving the toxicological risk assessment (Pellizzer et al., 
2005). Quantitative gene expression analyses using real-time quantitative polymerase chain reaction (PCR) in the presence or absence of test chemicals is commonly used to detect the genetic modifications caused by these treatment (Bremer et al., 2001). However, previous studies have not comprehensively examined the effects of soy phytoestrogens mixture on $\mathrm{mEScs}$ or $\mathrm{mEB}$.

Genistein and diadzein are the principal isoflavones phytoestrogens that are found in soybean and other legumes (Delmonte et al., 2006). Normal range of total genistein concentration was measured as 0-4192 $\mathrm{nM}$ with a mean of $501.9 \mathrm{nM}$ from the serum of Japanese women (Setchell et al., 2011). Isoflavones are structurally similar to estrogen. Moreover; genis-tein and diadzein can bind with and transactivate estrogen receptors (ER) (Sakamoto, 2009).

There were no previous studies evaluating the genetic/epigenetic changes that induced during the differentiation of mouse EScs as a result of exposure to these isoflavones either singly or in a combined treatment. Therefore, the current study aimed to shed light on the developmental alterations that occur following either single exposure or co-exposure of soy isoflavones (Genistein and Diadzein ) on the differentiation and proliferation capacities of the
$\mathrm{mEScs}$ in order to understand the effects and mechanism of phytoestrogen treatments on the early embryonic development. This in turn may provide useful insights into the expected risks to human populations and to regulate the use of such compounds to reduce the risk of the developmental anomalies.

\section{Materials and methods Mouse Embryonic stem cells (mEScs) culture:}

ES-E14TG2a (ATCC $®$ CRL-1821 TM embryonic stem cells were cultured and maintained in embryonic stem (ES) medium with routinely changing the medium every day and subculture three times per week in a ratio of $1: 5$ as previously reported by (Nagy et al., 2003) in a humid atmosphere at $37^{\circ} \mathrm{C}$ and $5 \% \mathrm{CO}_{2}$. The culture medium consisted of Dulbecco's modified Eagle's medium (Gibco) supplemented with $10 \%$ fetal bovine serum (Sigma), $1 \mathrm{mM}$ non-essential amino acids (Gibco), $100 \mu \mathrm{M}$ 2-mercaptoethanol (Sigma), 100u/ml Penicillin-Strepto-mycin (Gibco), 1 mM Lglutamine (Gibco) and 1,000 U/ml LIF (Home-made) on gelatin coated dishes as described by (Ema et al., 2008).

In vitro Differentiation procedure (Embryoid bodies' formation):

The mEScs differentiation is induced by suspension culture where a single 
mouse embryonic stem cell multiplied and differentiated while suspended in a liquid ES medium without LIF (Leukemia inhibitory Factor) for 5 days until the formation of spheroid aggregations of cells representing the mouse embryoid bodies (mEBs).

\section{Chemicals exposure:}

Both Genistein (GEN) (Wako \# 44672-0) and diadzein (DIAD) (SigmaAldrich \#486-66-8) of $98.0 \%$ purity were dissolved in dimethyl sulfoxide (DMSO) (Wako\# DSL 6103) for further evaluation. Using of DMSO as a solvent control, the final DMSO concentration in all the samples was $0.01 \%(v / v)$. The mEScs were exposed to a single or combined low concentration $(10 \mu \mathrm{M})$ of genistein and /or diadzein as well as a single or combined high concentration of 50 $\mu \mathrm{M}$ of genistein and /or diadzein. Treatment of mES cells was done from day 0 to day 5 of in vitro differentiation and divided into 7 groups as following: GEN 10, GEN 50 with a concentration of 10 and $50 \mu \mathrm{M}$ of genistein, respectively; DIAD10, DIAD50 with a concentration of 10 and $50 \mu \mathrm{M}$ of diadzein, respectively; DIAD10+GEN10 in which a combination of $10 \mu \mathrm{M}$ of both phytoestrogens occurred; DIAD50+GEN50 in which a combination of $50 \mu \mathrm{M}$ of both phytoestrogens occurred; while the last group was the control one where
ES media only contained the solvent. All are done in triplicate in three separate experiments. Samples were collected at the end of day 5 of mEBs differentiation.

\section{Assessment of growth of the em- bryoid bodies:}

To assess the growth of the cultured embryoid bodies, photographs were taken using the BIOREVO Keyence bz-9000 inverted microscope and analyzed using the Keyence bz-9000 microscope analyzer software by measuring the diameters of the formed embryoid bodies.

Evaluation of the influence of these different treatments on the size of the embryoid bodies was done by two methods of analysis: (1) Mean evaluation of the diameter of all formed EBs and the obtained result was expressed as Mean \pm SE; (2) Because the EBs showed variation in size within the same group so, the diameter measurement was classified into 6 groups: $(20-39 \mu \mathrm{m}), \quad(40-59$ $\mu \mathrm{m}),(60-79 \mu \mathrm{m}),(80-99 \mu \mathrm{m}),(100-$ $119 \mu \mathrm{m})$ and $(120-160 \mu \mathrm{m})$ and the obtained result was expressed as \% from the totally formed Ebs. .

RNA isolation and cDNA formation.

Total RNA was isolated using ISOGEN reagent (Nippon gene co., Itd. Japan) according to the manufacturer's protocol. The first strand com- 
plementary DNA (cDNA) was synthesized from $1 \mu \mathrm{g}$ of total RNA using QuantiTect Rev. Transcription Kit (Qiagen) according to the manufacturer's protocol.

\section{Real time quantitative polymerase chain reaction (RT-QPCR)}

RT-qPCR analysis of gene expression profiles of ES cells was used to analyze the relative level of expression of specific mRNAs, the amount of cDNA was normalized to ßactin mRNA. Differentiation and stemness markers mRNAs at specific stages of ES cell differentiation were compared with that of the undifferentiated ES cells. Quantitative PCR was performed in Thermal Cycler Dice Real Time System (Takara Bio Inc., Otsu, Shiga, Japan) using SYBR P Premix EX Taq II (Takara Bio Inc.) The amplification program included an initial step at $95^{\circ} \mathrm{C}$ for 15 minutes, followed by 40 cycles of denaturation at $95^{\circ} \mathrm{C}$ for 15 seconds, annealing at $60^{\circ} \mathrm{C}$ for 20 seconds, and extension at $72^{\circ} \mathrm{C}$ for $30 \mathrm{sec}-$ onds. Data were normalized against the expression of $\beta$-actin gene. Primer sequences are listed in Table (1).

\section{Statistical analysis:}

Three independent experiments were conducted in each study, and the results were expressed as mean \pm SE. The statistical differences between the groups were determined by analysis of variance (one way ANOVA) using SPSS ${ }^{\circledR}$ software (Statistical Package for Social science, version 17.01, Illinois, USA). The statistical significance was set at $P<0.05$, while considered highly significant at $P<0.01$.

\section{Results}

\section{Effects of phytoestrogen treat- ment on Embryoid bodies (mEBs) formation}

The presence of phytoestrogens in the medium did not prevent mEBs formation, which appeared as spherical masses after culturing mouse stem cells in a suspension ES medium without LIF in all the experiment-tal groups (Fig 1.a). Evaluation of the effect of these different phytoestrogens treatments on the size of the formed embryoid bodies revealed the following:

\section{1.a Genistein (GEN) treatment}

There was a significant decrease $(P<0.05)$ in the size of the embryoid bodies exposed to low concentration of genistein (GEN 10) as compared with that of the control group (Fig 1a $\& b)$. It was noticed an increase in the percentage of moderate sized mEBs $(60-79 \mu \mathrm{m}$ in diameter) which represented about $45 \%$, while those measured $80-99 \mu \mathrm{m}$ represented about $30 \%$ and the embryoid bodies measuring $40-59 \mu \mathrm{m}$ were about $20 \%$ 
of the total EBs. In addition, about $5 \%$ of the EBs were extremely small or largely sized. These data were significantly differed from those of the control group (Fig.1c). While there was a highly significant $(P<0.01)$ decrease in the diameter values of the mEBs formed after exposure to high concentration of genistein (GEN 50) (Fig.1.b) with high percentage $(40 \%)$ of the EBs measured $60-79 \mu \mathrm{m}$, followed by those measured $40-59 \mu \mathrm{m}$ which represented about $30 \%$ and finally about $20 \%$ of the EBs measured $80-99 \mu \mathrm{m}$ in diameter (Fig 1C).

\section{Diadzein (DIAD) treatment}

A clear significant decrease in the size of mEBs was recorded following exposure to low concentration of diadzein (DIAD10) with significant increase in the percentage of the small sized mEBs compared with that of the control (Fig 1). On the other hand, there was a non-significant difference in the size of the embryoid bodies with higher concentration (DIAD50) (Fig 1b). Also, the percentage embryoid bodies' size in the low and high concentrations appeared more or less similar to that of the control (Fig 1c).

\section{2.a Combined treatment}

Exposure to a low concentration of both reagents (DIAD10+GEN10) showed non-significant difference either in the diameter means or even in the percentage of different mEBs sizes compared with that of the control group (Fig 1). However, a high significant difference $(P<0.01)$ in the diameter mean values as well as the percentage of different mEBs sizes were observed after exposure to a high combined concentration (DIAD50+ GEN50), when compared with that of the control (Fig 1). It was noticed the presence of $45 \%$ of $\mathrm{mEBs}$ with $60-79 \mu \mathrm{m}$ in diameter followed by about $40 \%$ in case of $40-59$ $\mu \mathrm{m}$ (Fig 1c).

\section{2.b Effects of phytoestrogen treatment on proliferation capac- ity in mEBs}

The expression level of the proliferation marker (Cdk6) was analyzed by qRT-PCR. Treatment with (GEN10), (DIAD50) and (DIAD10+GEN10) showed non-significant difference in the expression level of Cdk6 compared with that of the control. However, there was significant decrease $(P<0.05)$ in the expression level in case of (GEN50) and (DIAD10) treatments. On the other hand, exposure to a combination of the high concentration (DIAD50+GEN50) showed significantly $(P<0.05)$ higher expression of the proliferation marker Cdk6 (Fig 2). 


\section{Effects of phytoestrogen treat- ment on apoptosis in mEBs}

The mRNA level of Caspase-3 (apoptosis marker) showed significant $\quad(P<0.05) \quad$ increase after (GEN10), (GEN50), (DIAD10) and (DIAD50+ GEN50) treatments. While non-significant change occurred in the other treated groups (Fig 2).

\section{Effects of phytoestrogen treat- ment on pluripotency state in mEBs}

Pluripotent embryonic stem (ES) cells are characterized by their almost unlimited potential to self-renew and to differentiate into virtually any cell type of the organism (Schroeder et al., 2009). To determine the effect of phytoestrogens exposure on the pluripotency state while differentiation progresses, the expression levels of pluripotency-associated transcription factors were evaluated at the mRNA level.

\section{4.a Genistein (GEN) treatment}

The expression level of the transcription factors Oct4, Rex1 and Nanog were not affected by the low concentration treatment of genistein (Fig 3). However, in high concentration group (GEN 50), the levels of Rex1 and Oct4 were higher than that of the control group. On the other side, Nanog expression level was non-significantly changed in this group compared with their levels in the control (Fig 3).

J. Vet. Anat.

\section{4.b Diadzein (DIAD) treatment}

The group treated with low level of daidzein (DIAD10) showed non-significant difference in the expression level of these pluripotency transcription factors. While exposure to a high concentration (DIAD50) led to increase the expression levels of Rex 1 and Oct4 with non-significant change in case of Nanog expression levels (Fig 3).

\section{4.c Combined treatment}

Low concentration of combined (DIAD10+GEN10) exposure did not affect the level of Nanog but led to a high significant overexpression of Rex1 (Fig 3). Exposure to high concentration combination (DIAD50+ GEN50) led to a high significant increase in the mRNA levels of Oct4 and Rex1. In contrast, the level of Nanog mRNA was significantly decreased than that of the control group (Fig 3).

\section{Effects of phytoestrogen treat- ment on differentiation capac- ity in mEBs}

To detect the effect of phytoestrogens on the differentiation capacity of the mEBs, the expression of Gata 6, Foxa 2, Fgf5 and Sox 17 were examined as mesoendoderm markers while Nestin, Pax 6 and Sox 1 were chosen as ectoderm markers.

\section{5.a Genistein (GEN) treatment}

Treatment of the formed mEBs with a low concentration of genistein 
(GEN 10) showed non-significant difference either in the mesoendoderm or ectoderm markers (Figs 4\&5). In case of (GEN50) treated group, highly significant overexpression of Foxa2 (100 folds) was detected (Fig 5). Additionally, significant high expression was detected in Gata6 level (9 folds) together with high level of Sox 17 (2 folds) and Fgf5 (5 folds) (Fig 5). In this connection, the ectoderm markers Sox 1 and Pax 6 in this group were significantly increased (14 folds) com-pared with that of the control while Nestin expression level remained non-significantly changed (Fig 4).

\section{5. b Diadzein (DIAD) treatment}

The expression levels of mesoendoderm and ectoderm markers remained non-significantly changed in (DIAD10) group (Figs 4\&5). However, mEBs treated with (DIAD50) showed significantly high expression Foxa2 and Sox17 levels. While Fgf5 and Gata6 mRNA levels remained non-significantly affected (Fig 5). Moreover, highly significantly overexpression of Sox 1 and Pax6 was noticed in this group with non-affected level of Nestin mRNA (Fig 4).

\section{5.c Combined treatment}

The mRNA levels for ectoderm markers and mesoendoderm differentiation markers (Gata6, Fgf5, Foxa2 and Sox17) were unaffected during treatment with (DIAD10+GEN10) (Figs 4\&5). On the other hand, treatment with (DIAD50+GEN50) only led to a significant high expression of Fgf5 while the other mesoendoderm markers were not affected (Fig 5). A highly significant overexpression of Sox 1 and Pax6 mRNA were detected but the level of Nestin remained unaffected (Fig 4).

\section{Effects of phytoestrogen treat- ment on Esrrb Expression level.}

A clear decrease of the Esrrb expression level was noticed in case of (GEN50), (DIAD50) (DIAD10+ GEN 10) and (DIAD50 + GEN50), while non-significant effects appeared in the other treated groups (Fig 6).

\section{Discussion}

Effects of bioactive phytochemicals, including phytoestrogens, appeared to be acting at several levels in the cells and trigger at the same time several pathways (Spagnuolo et al., 2015). Our entire findings confirmed that exposure of soy phytoestrogens manifested effects in different ways on the stem cells proliferation, differentiation and apoptosis.

The results revealed a marked decrease in the size of the mEBs after exposure to a higher concentration $(50 \mu \mathrm{M})$ of genistein either alone or in a combination with $50 \mu \mathrm{M}$ of diadzein. Also, an exposure to a low 
concentration of genistein or daidzein alone decreased the mEBs size whereas surprisingly, after a low concentration co-treatment of both phytoestrogens, the inhibitory effect on the mEBs size disappeared suggesting that both phytoestrogens might antagonist each other when combined at low concentration but the effect was emphasized at a high concentration co-treatment.

Our results also suggested that exposure to both high and low concentrations of genistein as well as only low concentration of daidzein increased the cell death on the mouse Embryoid bodies that detected by overexpression of Caspase-3 gene (apoptosis marker) and down regulation of Cdk6 gene (proliferation marker). The same effects on the cell death and proliferation were recorded by (Kong et al., 2013) after genistein treatment of the mouse embryonic stem cells, midbrain cell culture study (Xiaoa et al., 2011) as well as in studying the effect of genistein and daidzein on the cultured cells of breast, ovarian, lung and prostate cancers, leukemia and lymphoma (Gercel-Taylor et al., 2004; Liu et al., 2013; Yanagihara et al., 2013; Huang et al., 2014). At the same time, genotoxic adverse effects of genistein including apoptosis, cell growth inhibition and DNA damage were reported in vitro in experimental animals (Ramos, 2007).
Also, other studies confirmed that daidzein can induce apoptotic and antiproliferative effects in a concentration and time-dependent manner in different cancer cell lines (GercelTaylor et al., 2004; Choi and Kim, 2008; Jin et al., 2010).

Interestingly, the co-treatment of low concentrations of both genistein and diadzein in the present study showed no change in the size, apoptosis or proliferation of mEBs compared with those of the non-treated group. On the other hand, co-treatment with high concentrations of both isoflavones decreased the mEBs size and caused over expression of either apoptosis or proliferation markers. These results might suggest that the use of combined isoflavones had variable effects on the newly formed mEBs as they antagonized each other at low concentrations while agonisted (synergisted) each other at high concentrations thus, the inhibitory effect on mEBs growth might be caused by increasing apoptosis level together with decreasing the cell proliferation.

From another point of view, the expression level of Cdk6 might not only be considered an indicator of phytoestrogens effect on the cell cycle and proliferation but might be also influenced by their effect on the stem cell differentiation as the activity of 
Cdk6 is essential to define the proliferative and differentiating capacity of multipotent stem cells (Pauklin and Vallier, 2013). In addition, Cdk6 is activated immediately upon differentiation of mouse embryonic stem cell (Bryja et al., 2008), thus in our point of view, examination of the pluripotency and differentiation markers was essential to elucidate the mechanism by which single or combination of phytoestrogen treatments affected the mEBs formation.

Both $\mathrm{mES}$ cells and in vivo cells expressed key pluripotency factors, such as Nanog, Oct4 (Young, 2011). In ESCs, Oct4 (the POU transcription factor encoded by Pou5f1) and Nanog (the natural killer-2 class homeobox transcription factor) were known as key regulators based on their unique expression pattern in ESCs (Chambers and Smith, 2004). In addition, $\operatorname{Rex} 1$ (zfp42) is a zinc finger protein that expressed primarily in undifferentiated stem cells, both in embryo and adult mouse (Scotland, 2009).

Early differentiation of pluripotent mouse embryonic stem (mES) cells, which are derived from the inner cell mass of the pre-implantation (blastocyst) stage embryo summarized various aspects of in vivo germ layer differentiation (Keller, 2005). During this period, the balance between pluripotency and differentiation was very important where in our study, the expression level of pluripotent and differentiation markers of the formed mEBs were examined carefully using the RT-qPCR analysis and it was found that treatment with a low concentration of genistein or daidzein did not affect the pleuripotancy and differentiation markers in the developing mEBs indicating that exposure to a low level of an individual soy isoflavone might have no effect in the early stage of the embryonic development either on the stem cell markers or the differentiation markers. Surprisingly, a combination of low dose of both compounds led to marked up regulation in the expression level of pleuripotancy markers Oct4 and Rex1 while Nanog and differentiation markers expression levels remained unchanged. These data suggested that low concentration co-treatment of phytoestrogens didn't affect the differentiation capacity of the mouse embryonic stem cells but accompanied with increase in pleuripotancy markers Oct4 and Rex 1 expression. Furthermore, the high concentration of a single treatment of soy phytoestrogen cau-sed over expression of the stem cell factors Oct4 and Rex1, whereas expression of Nanog remained unchanged together with extreme over expression in the levels of ectoderm markers and mesoendoderm markers. The current data also 
revealed that a high dose treatment of individual phytoestrogen increase the differentiation capacity of the developing $\mathrm{mEB}$ in comparison with that of the control group.

Interestingly, when the mEBs were exposed to a mixture of high concentration of genistein and daidzein, the levels of the mesoendoderm markers appeared to be at levels approaching those of the control group. Whereas, levels of Oct4 and Rex1 remained elevated with decreased level of Nanog, the findings that agreed with those of (Regenbrecht et al., 2008; Van den Berg et al., 2008). Additionally, up regulation of the ectoderm markers detected in the present study indicated that $\mathrm{mEB}$ cells differentiation capacity were directed toward the ectoderm cell lines.

From the previous findings, it was concluded that phytoestrogen treatments might increase the differentiation ability of the mouse stem cells more than that in the control condition depending on the concentration and also on whether single or cotreatment of these soy phytoestrogens were used. This was confirmed by increase of the differentiation markers of the different germ layers or only ectoderm markers that accompanied by elevation of Oct4 and Rex1 transcription factors and in some cases with down regulation of Nanog in comparison with the control condition.
Regarding gene expression profile during mouse stem cells differentiation, the recent results confirmed those of (Mitsui et al, 2008) where Oct4 levels were tightly regulated by the stem cells and precise levels of Oct4 must be sustained for the maintenance of pluripotency, however, down-regulation of the Oct4 protein led to a loss of maintenance of ES cells pluripotency. Also, overexpression of Oct4 induced differentiation in ES cells as mentioned by (Mitsui, 2008). Expression levels of Oct4 and Rex1 increased significantly in the early stages of mouse embryonic stem cells differentiation (Sene, 2007). On other hand, Loss or deficiency of Nanog results in ES cell differentiation with up regulation of ectoderm marker genes (Scerbo, 2012).

Moreover, the expression level of Esrrb was examined for more clear understanding of possible pathway of these phytoestrogens on the $m E S c s$ proliferation and differentiation capacities. Previous studies mentioned that Esrrb expressed exclusively in mouse ES cells and during process of embryoid body formation, where they have role in trophoblast differentiation and placenta formation (Carter, 2007). ESRRB was able to sustain EScs pluripotency and self-renewal in the absence of LIF (Zhang, 2008) and also can be co-immunoprecipitated 
as part of the protein complex associated with NANOG in ES cells (Wang, 2006). Moreover, Esrrb knockdown induced ES cell differentiation (Wang, 2006). From another point of view, ESRRB shared significant homology with the estrogen receptor and belonged to the superfamily of nuclear receptors, that are classified as orphan receptors because they bind to DNA and are transcriptionally active in the absence of identified exogenous ligand (Giguere et al., 1988; Schuff et al., 2012). While, Phytoestrogens can interact with the classical estrogen receptors, $E R \alpha$ and $E R \beta$ by mediating many of their downstream actions through agonist or antagonist activity (Lorand, 2010) and because estrogenrelated receptors (ERRs) are involved in similar estrogen receptor (ER). So, these soy phytoestrogens could bind to and resulted in increased or decreased activity of ESRRB protein (Suetsugi et al., 2003). Our findings showed no change in the Esrrb expression level in case of single low concentration treatment of genistein, daidzein, however, a significant change occurred after low concentration cotreatment of both compounds. Moreover, Esrrb expression level appeared to be also markedly down regulated after high concentration treatment of single or combined phytoestrogen. These RT-qPCR data suggested that a single high concentration capacity of phytoestrogen increased the differentiation capacity of the developing mEBs through antagonizing ESRRB receptors. While co-treatment of high concentration induced the differentiation of the mouse embryonic stem cells toward the ectoderm through down regulation of Esrrb and Nanog together with up regulation of Cdk6, confirming what stated (Scerbo, 2012 Schuff et al., 2012).

In conclusion, it was firstly observed that down regulation of Esrrb is associated with up-regulation of pluripotent marker genes including Oct4 and $R e x 1$ during exposure to a low concentration of a mixture of soy isoflavones or a high concentration of a single compound, while Nanog down regulation and Cdk6 up regulation were observed upon high concentration of soy isoflavones mixture treatment in mEScs and mEBs. These findings enhanced our understanding of mechanism of soy phytoestrogen exposure effects on the embryonic development.

Finally, and according to the current results, the exposure of soy phytoestrogens either alone or in a combined manner caused some adverse effects during the early development of the mouse embryo. Therefore, the biological significance of these find- 
ings regarding the influences of phytoestrogens on the proliferation and differentiation capacities of the mEScs should be taken into consideration.

\section{Acknowledgments}

This work was carried out with the support of the Joint Mission Program from Ministry of Higher Education and Scientific Research between Suez Canal University, Arabic Republic of Egypt and short exchange research student program, Tsukuba University, Japan.

\section{Potential conflict of interest}

The authors have no conflicting financial interest.

\section{References}

Barouki R.; Gluckman P.D.; Grand-jean P.; Hanson M.\& Heindel J.J. (2012): Developmental origins of non-communicable disease: implications for research and public health. Environmental Health, 11(1), 42.

Bremer S.; Worth A.P.; Paparella M.; Bigot K.; Kolossov E.; Fleischmann B.K. (2001): Establishment of an in vitro reporter gene assay for developmental cardiac toxicity. Toxicol In Vitro, 15:215-23.

\section{Bryja V., Pacherník J., Vondráček}

J., Souček K., Čajánek L., Horvath V., Holubcová Z., Dvořák P., Hampl A. (2008): Lineage specific composition of cyclin DCDK4/CDK6-p27 complexes reveals distinct functions of CDK4, CDK6 and individual D-type cyclins in differentiating cells of embryonic origin. Cell Prolif, Dec; 41(6): 875893.

Carter A.M. (2007): Animal models of human placentation -a review Placenta; Cell Prolif 41(6): 875-893.

Chambers I. and Smith A. (2004): Self-renewal of teratocarcinoma and embryonic stem cells. Oncogene, 23, 7150-7160.

Choi E.J., Kim G.H. (2008): Daidzein causes cell cycle arrest at the $\mathrm{G} 1$ and $\mathrm{G} 2 / \mathrm{M}$ phases in human breast cancer MCF-7and MDA-MB453 cells, dd Phytomedicine, vol. 15 :683-690.

Delmonte P.; Perry J.; Rader J.I. (2006): Determination of isoflavones in dietary supplements containing soy, Red Clover and kudzu: extraction followed by basic or acid hydrolysis. J Chromatogr A, 1107: 59-69.

Ema M., Mori D., Niwa H., Hasegawa Y., Yamanaka Y., Hitoshi S. (2008): Kruppel-like factor 5 is essential for blastocyst development 
and the normal self-renewal of mouse ESCs. Cell Stem Cell, 3(5): 555-67.

Gercel-Taylor C., Feitelson A.K., Taylor D.D. (2004): Inhibitory effect of genistein and daidzein on ovarian cancer cell growth. Anticancer Res, 24(2B): 795-800.

Giguere V., Yang N., Segui P. and Evans R. M. (1988): Identification of a new class of steroid hormone receptors, Nature, 331, 91-94

Huang W., Wan C., Luo Q., Huang Z. (2014): Genistein-inhibited cancer stem cell-like properties and reduced chemo resistance of gastric cancer. Int J Mol Sci, 15:3432-43.

Jin S., Zhang Q.Y., Kang X.M., Wang J.X., Zhao W.H. (2010): Daidzein induces MCF-7 breast cancer cell apoptosis via the mitochondrial pathway. Ann Oncol; 21 (2): 263268.

Keller G. (2005): Embryonic stem cell differentiation: emergence of a new era in biology and medicine. Genes Dev, 19: 1129-1155.

Kong D., Xing L., Liu R., Jiang J., Wang W., Shang L., Wei X. and Hao W. (2013): Individual and combined developmental toxicity assessment of bisphenol $A$ and genistein using the embryonic stem cell test in vitro Food Chem. Toxicol, 60, pp. 497-505

Liu Y.L., Zhang G.Q., Yang Y., Zhang C.Y., Fu R.X., Yang Y.M. (2013): Genistein induces G2/M arrest in gastric cancer cells by increasing the tumor suppressor PTEN expression. Nutr Cancer, 65:1034-41.

Lorand T., Vigh E. and Garai J. (2010): Hormonal action of plant derived and anthropogenic non-steroidal estrogenic compounds: phytoestrogens and xenoestrogens. Curr Med Chem, 17:3542-3574.

Martin G.R. (1981): Isolation of a pluripotent cell line from early mouse embryos cultured in medium conditioned by teratocarcinoma stem cells. In Proceedings of the National Academy of Sciences of the United States of America; 78(12):76347638 .

Mitsui K., Tokuzawa Y., Itoh H., Segawa K., Murakami M., Takahashi K., Maruyama M., Maeda M., Yamanaka S. (2008): The homeoprotein Nanog is required for maintenance of pluripotency in mouse epiblast and ES cells. Cell 113:631642.

Nagy A.; Perrimon N, Sandmeyer 
S, Plasterk R. (2003): Tailoring the genome: the power of genetic approaches. Nat Genet, 33 Suppl:27684.

Pauklin S., Vallier L. (2013): The Cell-Cycle State of Stem Cells Determines Cell Fate Propensity. Cell, 155: 135-147.

Pellizzer C.; Bremer S.; and Hartung T. (2005): Developmental toxicity testing from animal towards embryonic stem cells, 22, 47-57.

Ramos S. (2007): Effects of dietary flavonoids on apoptotic pathways related to cancer chemoprevention. J Nutr Biochem, 18:427-42.

Regenbrecht C.R, Jung M., Lehrach $\mathrm{H}$. and Adjaye J. (2008): The molecular basis of genistein-induced mitotic arrest and exit of self-renewal in embryonal carcinoma and primary cancer cell lines. BMC Med Genomics, 1: 49.

Sakamoto T.; Horiguchi H.; Oguma E.; Kayama F. (2009): Effects of diverse dietary phytoestrogens on cell growth, cell cycle and apoptosis in estrogen-receptor-positive breast can-cer cells. J Nutr Biochem, (9):856-64.

Scerbo P., Girardot F., Vivien C., Markov G.V., Luxardi G, Demeneix
B., Kodjabachian L., Coen L. (2012): Ventx factors function as nanog-like guardians of developmental potential in xenopus. PLoS One, 7 (5): e36855.

Schroeder I., Wiese-Rischke C., Truong, T., Rolletschek, A., Wobus A. (2009): Differentiation Analysis of Pluripotent Mouse Embryonic Stem (ES) Cells In Vitro. Methods in molecular biology (Clifton, N.J.). 530. 219-50.

Schuff M., Siegel D., Philipp M., Bundschu K., Heymann N., Donow C., Knöchel W. (2012): Characterization of Danio rerio Nanog and functional comparison to Xenopus Vents. Stem Cells Dev, 21 (8): 12251238.

Scotland K.B., Chen S., Sylvester R., Gudas L. J. (2009): Analysis of Rex1 (zfp42) function in embryonic stem cell differentiation. Dev Dyn, 238(8):1863-77.

Sene K.H., Porter C.J., Palidwor G., Perez-Iratxeta C., Muro E.M., Camp-bell P.A., Andrade-Navarro M.A. (2007): Gene function in early mouse embryonic stem cell differentiation. BMC Genomics, 8, 85.

Setchell K.D.; Brown N.M.; Zhao X. (2011): Soy isoflavone phase II metabolism differs between rodents and 
humans: implications for the effect on breast cancer risk. Am J Clin Nutr, 94: 1284-1294.

Spagnuolo C, Russo G.L., Orhan I.E., Habtemariam S., Daglia M., Sureda A., Nabavi S.F., Devi K.P., Loizzo M.R., Tundis R., and Nabavi S.M. (2015): Genistein and Cancer: Current Status, Challenges, and Future Directions Adv Nutr, vol. 6: 408419.

Suetsugi M., Su L., Karlsberg K., Yuan Y.C., Chen S. (2003): Flavone and isoflavone phytoestrogens are agonists of estrogen-related receptors. Mol Cancer Res, 1, 981-991.

Van den Berg D.L.C., Zhang W., Yates A., Engelen E., Takacs K., Bezstarosti K., Demmers J., Chambers I., Poot R.A. (2008): Estrogen-related receptor beta interacts with Oct4 to positively regulate Nanog gene expression. Mol Cell Biol, 28: 5986-5995.

Vandenberg L.N.; Colborn T.; Hayes T.B.; Heindel J.J.; Jacobs D.R.; Lee D.H.; Shioda T.; Soto A.M.; Vom Saal F.S.; Welshons W.V.; Zoeller R.T.; Myers J.P. (2012): Hormones and endocrine-disrupting chemicals: low-dose effects and nonmonotonic dose responses. Endocr Rev, 33 (3): 378-455.
Wang J., Rao S., Chu J., Shen X., Levasseur D.N., Theunissen T.W. and Orkin S.H. A (2006): protein interaction network for pluripotency of embryonic stem cells. Nature, 444, 364-368.

Xiaoa Y, Liua R, Xinga L, Xub Y, Shanga L, Haoa. (2011): Combined developmental toxicity of bisphenol $A$ and genistein in micromass cultures of rat embryonic limb bud and midbrain cells, Toxicology in Vitro, Volume 25, Issue 1, 153-159.

Yanagihara K., Takigahira M., Mihara K., Kubo T., Morimoto C., Morita Y., Terawaki K., Uezono Y., Seyama T. (2013): Inhibitory effects of isoflavones on tumor growth and cachexia in newly established cachectic mouse models carrying human stomach cancers. Nutr Cancer 65:578-89.

Young R.A. (2011): Control of Embryonic Stem Cell State. Cell, 144(6):940-954.

Zandstra P.W.; Le H.V, Daley G.Q.; Griffith L.G.; Lauffenburge D.A. (2000): Leukemia inhibitory factor (LIF) concentration modulates embryonic stem cell self-renewal and differentiation independently of proliferation. Biotechnol. Bioeng, 69: 607-617. 
Influence of soybean phytoestrogens on mouse stem cells

Zhang X., Zhang J., Wang T.,

Esteban M.A., Pei D. (2008): Esrrb

activates Oct4 transcription and sus-

tains self-renewal and pluripotency

in embryonic stem cells. J Biol

Chem, 19; 283(51): 35825-33.

\section{Corresponding author}

Walaa Abdelwahab Basha (Basha, W.A.)

E-mail: walaaanatmy85@yahoo.com

Mailing address: 1-1-1 Tennodai, Tsukuba, Ibaraki, Japan

Laboratory Animal Resource Center

University of Tsukuba

Table (1): List of RT-qPCR primer sequences

\begin{tabular}{lll}
\hline Gene & Primer Sequence (Forward) & Primer Sequence (Reverse) \\
\hline -actin & GTGTAAAACGCAGCTCAGTAACAGT & CTGAGCGCAATACTCTGTGTG \\
Oct3/4 & TATTGAGTATTCCAACGAGAAGAG & CTCAGGAAAGGGACTGAGTAGAGT \\
Nanog & CTTTCACCTATTAAGGTGCTTGC & TGGCATCGGTTCATCATGGTAC \\
Esrrb & ATGAATGAGATCACCAAACG & GTTCAGGTAGGGGCTGTTCTC \\
Rex 1 & TCCATGGCATAGTTCCAACAG & TAACTGATTTTCTGCCGTATGC \\
Foxa2 & AGACTACTGCTTCTCAAGACATCTG & CCTCTTTTCAACATCAGTACAACCC \\
Sox17 & CAGTATCTGCCCTTTGTGATAAGC & GTAGTTGCAATAGTAGACCGCTGAG \\
Pax6 & ATGGAGAAAGAAGAGAACTGAGG & CTGGTAGACACTGGTACTGAAGC \\
Fgf5 & ACCCACTTCCTACCCAGGTT & AGTTGTTTCCCACAAGGCCA \\
Nestin & CCTCAACCCTCACCACTCTATTTTA & TTCTTTACAAGTTCCCAGATTTGCC \\
Sox1 & ACAGCGTTTTCTCGGCTTCG & GAGCTGGCGGGAAGAAACC \\
Casps & GGGCGTGTTTCTGTTTTGTT & TGCATTGCTAGGCAGTGGTA \\
$\mathbf{3}$ Colk6 & GCTTCGTGGCTCTGAAGCGCG & TGGTTTCTGTGGGTACGCCGG \\
\hline
\end{tabular}


(a)
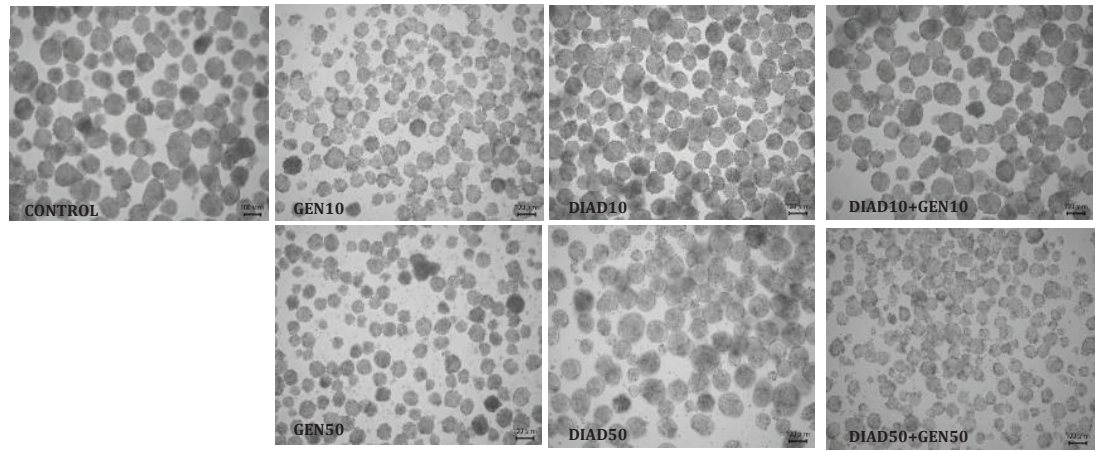

(b)

(c)
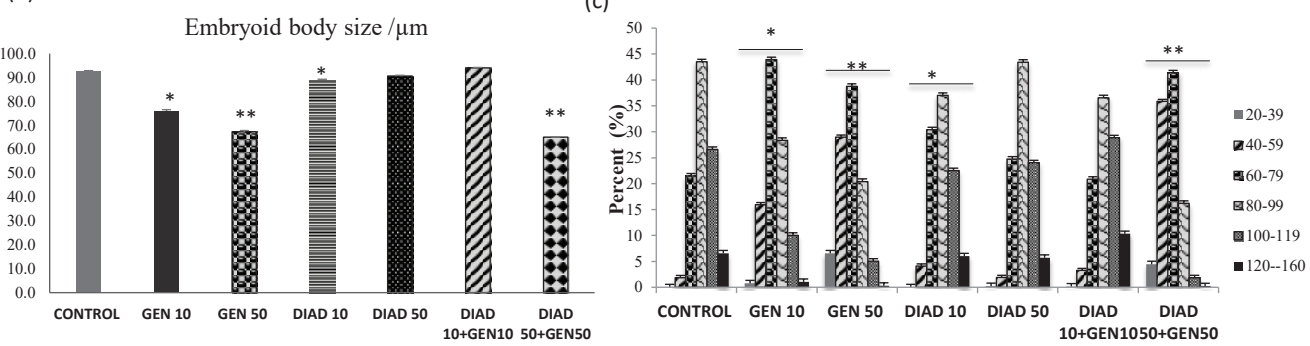

Fig. (1): Showing the effect of phytoestrogens treatment on mouse Embryoid bodies (mEBs) formation.

(a) The morphological appearance of mouse EBs in the suspension culture Scale bars: 100

$\mu \mathrm{m}$.

(b) The diameters of mEBs were used as an indication of their growth under the different treatment conditions compared with the control group; values were represented as means \pm SE of the three independent experiments.

(c) Categories of different mEBs size were recorded; data were presented as percentage of the total EBs within each group in the three independent experiments. $\left({ }^{*} \mathrm{P}<0.05\right.$, ${ }^{* *} \mathrm{P}<0.01$ 

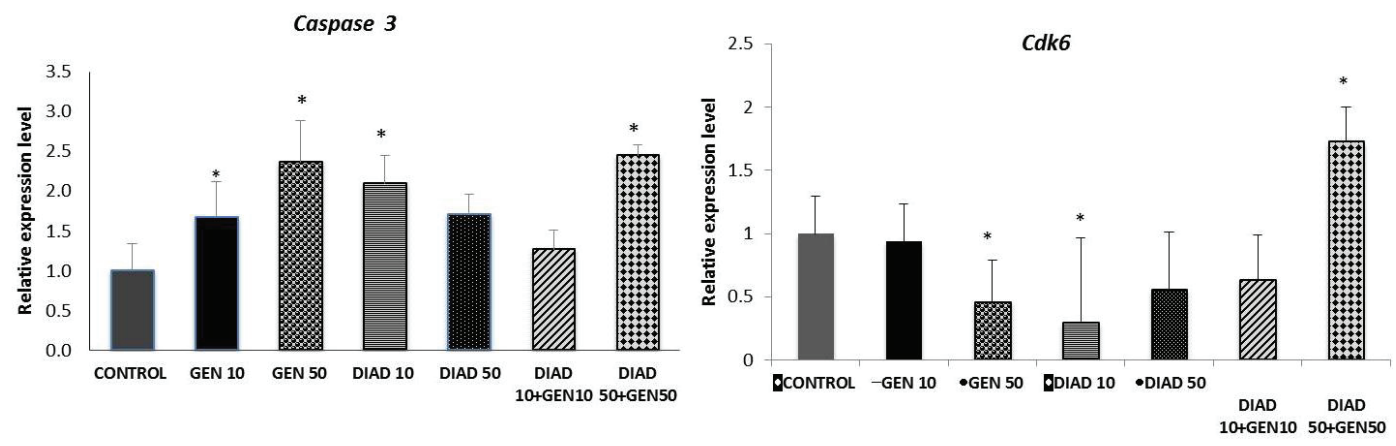

Fig. (2): Effects of phytoestrogen treatment on proliferation capacity and apoptosis of the mEBs. Expression level of Caspase-3, as apoptosis marker, was significantly up regulated in (GEN10), (GEN50), (DIAD10) and (DIAD50+GEN50) groups while, expression level of $C d k 6$, as proliferation marker, showed significantly down regulation in the same groups. The mean $\pm S E$ of the three independent experiments were shown using housekeeping $\beta$-actin as an internal control $\left({ }^{*} p<0.05\right)$

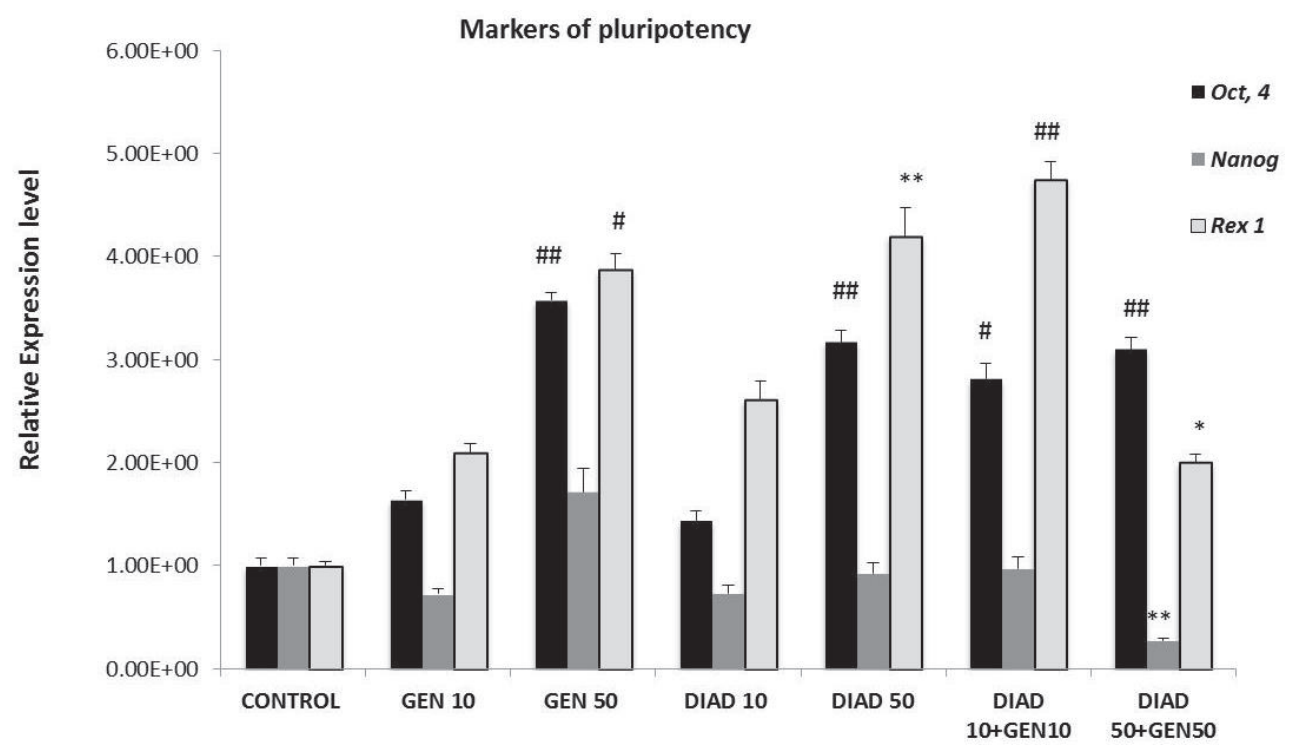

Fig. (3): Effects of phytoestrogen treatment on pluripotency state in mEBs. Changes in the expression level of pluripotency marker, Oct 4 and Rex1 were up regulated in the group treated with (GEN50), (DIAD50), (DIAD10+GEN10) and (DIAD50+GEN50), while Nanog expression only down regulated in (DIAD50 + GEN50). The means \pm SE of the three independent experiments were shown with using housekeeping $\beta$-actin as an internal control $\left({ }^{*} p<0.05,{ }^{* *} p<0.01, \# p<0.001, \# p<0.0001\right)$. 


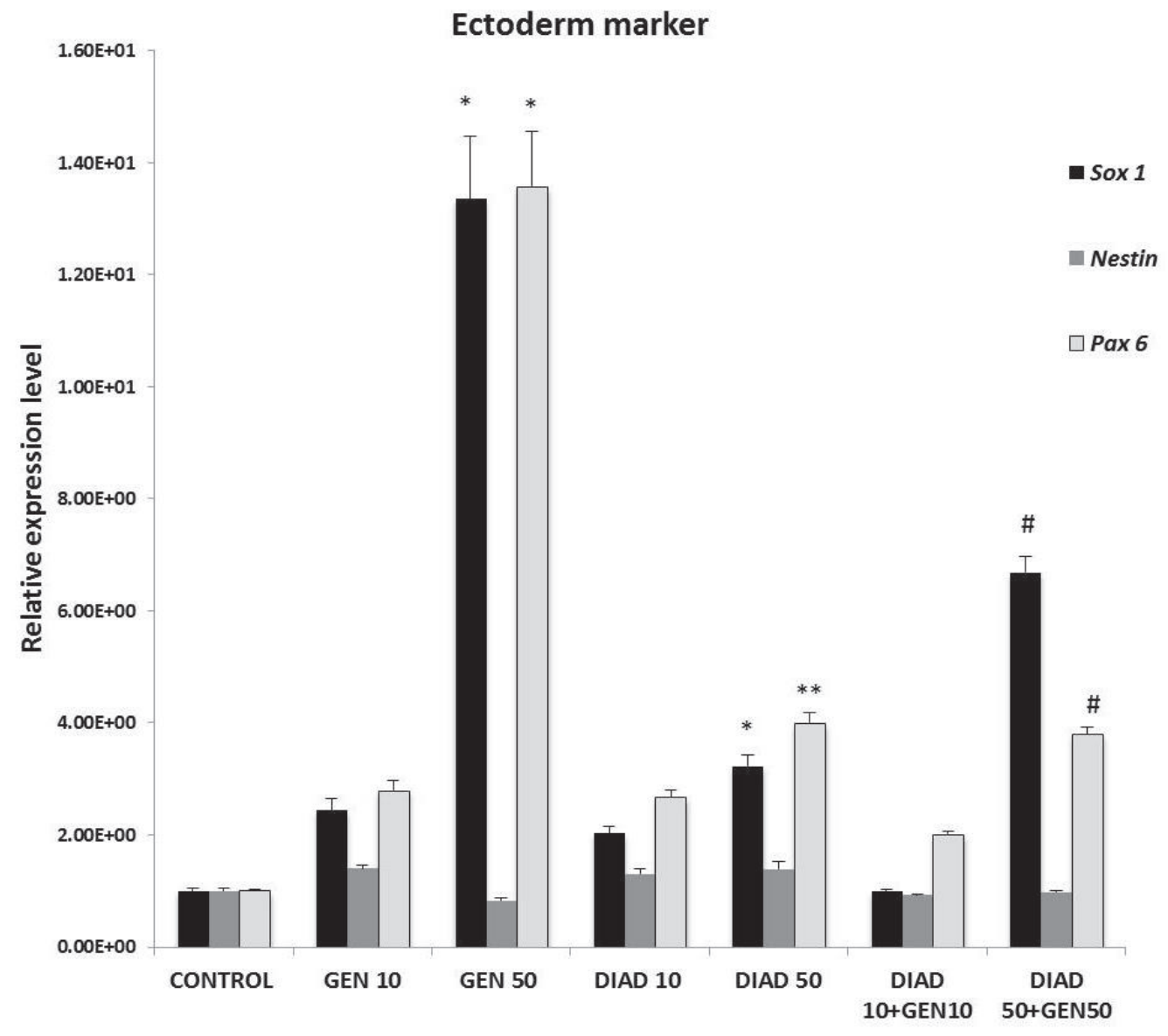

Fig. (4): Showing the changes in the expression level of ectoderm markers following phytoestrogen treatment. Note that Sox 1 and Pax 6 were significantly overexpressed in (GEN 50), (DIAD50) and (DIAD50 + GEN50) treated groups while Nestin expression was not affected by phytoestrogen treatment. Data were presented as the means \pm SE of the three independent experiments using housekeeping $\beta$-actin as an internal control $\left({ }^{*} p<0.05,{ }^{* *} p<0.01, \# p<0.001\right)$. 


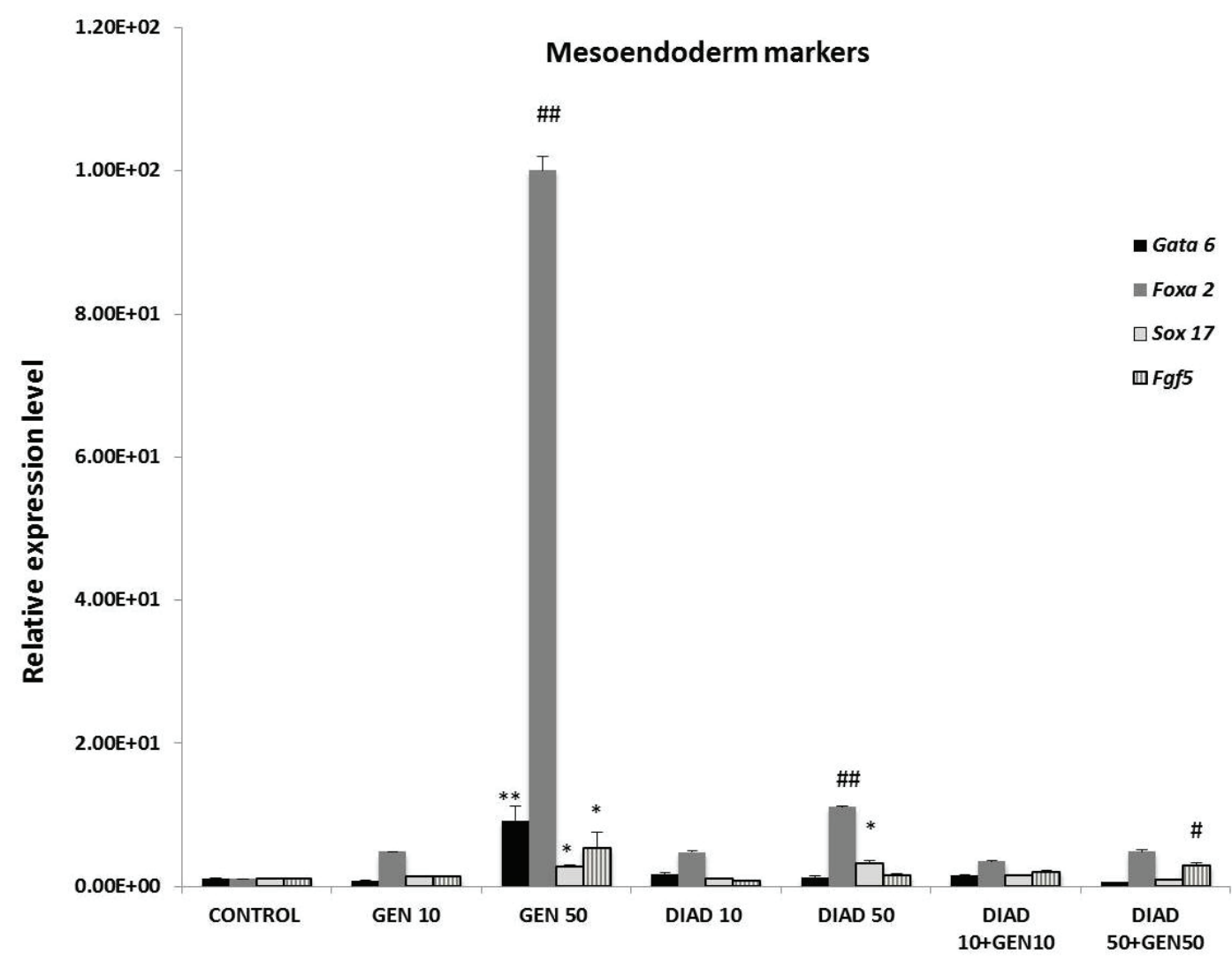

Fig. (5): Showing the Changes in the expression level of mesoendoderm markers following phytoestrogen treatment. Note that Foxa2 was significantly overexpressed in (GEN 50 ) and (DIAD50) treated groups while treatment with (GEN10), (DIAD10) did not affect the expression level of these markers compared with the control group. Data were presented as the mean \pm SE of the three independent experiments using housekeeping $\beta$ actin as an internal control $\left({ }^{*} p<0.05,{ }^{* *} p<0.01, \# p<0.001, \# p<0.0001\right)$. 


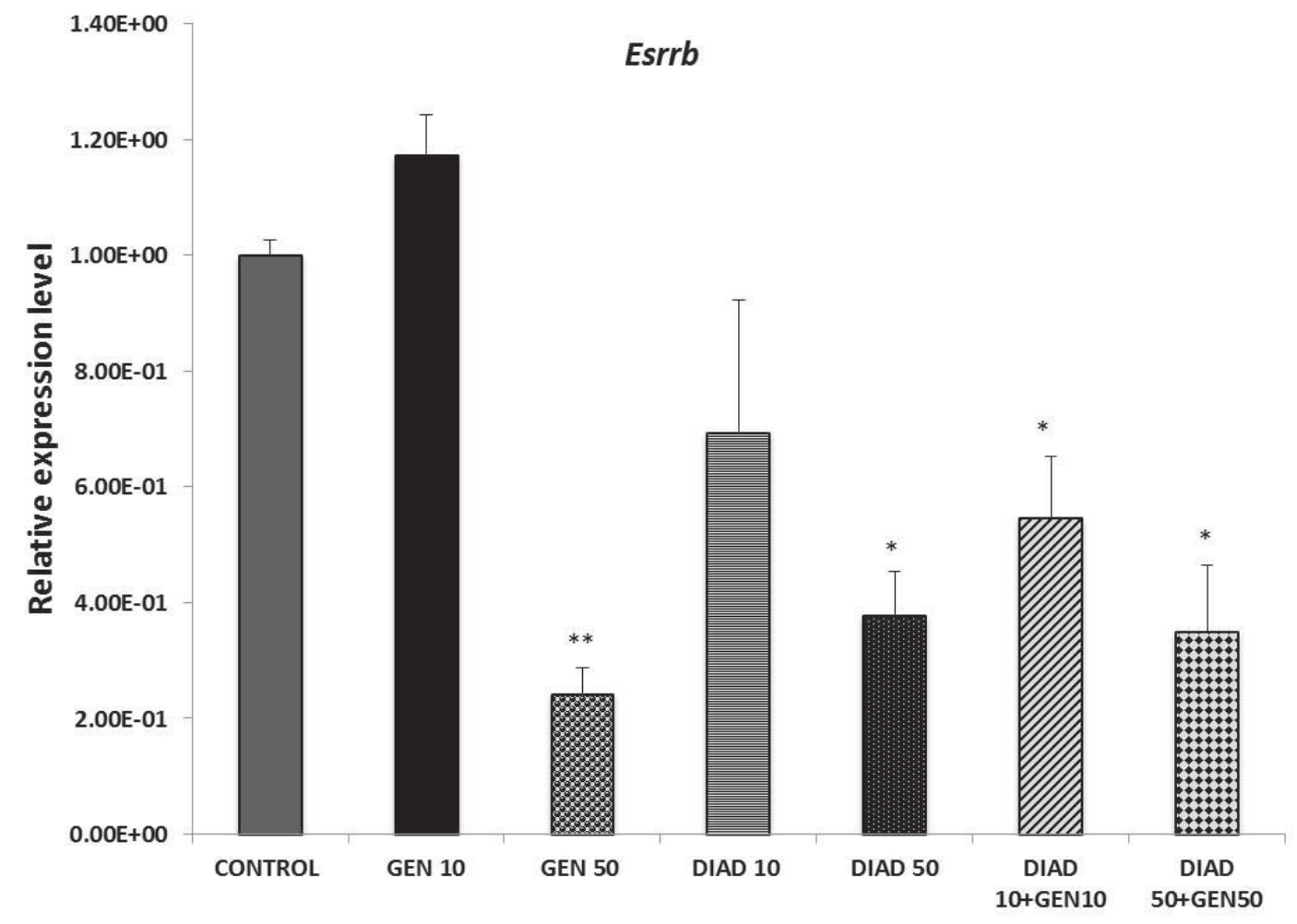

Fig. (6): Effects of phytoestrogen treatment on Esrrb Expression level. Down regulation of Esrrb expression was detected in (GEN50), (DIAD50), (DIAD10+GEN10) and (DIAD50+GEN50) treated groups while the other groups showing non-significant change on the expression level of Esrrb. Data were presented as the means $\pm S E$ of the three independent experiments using housekeeping $\beta$-actin as an internal control $\left({ }^{*} p<0.05\right.$, ** $\mathrm{p}<0.01$ ). 